\title{
Effects of rumen-protected choline supplied at different dietary energy levels on growth performance and meat quality of fattening goats
}

\author{
Y.L. Tu', , K. Zhang ${ }^{1,2}$, Y.F. Bai ${ }^{1,2,4}$, L.P. Gao ${ }^{1,2}$ and W. Hong ${ }^{3}$ \\ 1 Jiangsu Academy of Agricultural Sciences, Institute of Animal Science, Nanjing 210014, Jiangsu, China \\ ${ }^{2}$ Ministry of Agriculture, Key Laboratory of Crop and Livestock Integrated Farming, Nanjing 210014, Jiangsu, China \\ ${ }^{3}$ Shanghai Menon Animal Nutrition Technology Co., LTD., Shanghai 200157, Shanghai, China
}

KEY WORDS: choline, energy level, goats, meat quality, supplementation, biochemical indices

Received: 25 February 2020

Revised: $\quad 13$ May 2020

Accepted: 1 September 2020

${ }^{4}$ Corresponding author:

e-mail: baisubmit2020@126.com

\begin{abstract}
The effects of rumen-protected choline (RPC) supplementation in different energy level diets on growth performance and meat quality of fattening goats were examined in this study. In total, 40 six-month-old Cashmere goats (initial body weight $24.30 \pm 2.64 \mathrm{~kg}$ ) were randomly assigned to four treatments: two different digestible energy (DE) levels (11.94 and $10.89 \mathrm{MJ} / \mathrm{kg}$ ) and RPC supplement levels ( 0 and $0.2 \%$ ). The experiment lasted for 70 days, with the first 10 days of acclimation and next 60 days of formal experimental period. It was shown that RPC supplementation in a low-energy diet improved the overall average daily feed intake, and the interaction effect of RPC $\mathrm{DE}$ was highly significant between days 20 to 60 of the experiment. The overall average daily gain and feed to gain indices were not significantly affected by RPC supplementation at any energy level; however, RPC supplementation in a lowenergy diet tended to improve these indices. There were no significant effects of RPC $\times D E$ on slaughter performance, meat quality or serum biochemical indices; however, RPC supplementation resulted in lower drip loss, cook loss and shearing force, with a tendency to improve meat quality. Another positive effect of RPC supplementation was the enhancement of serum albumin and the reduction of serum urea nitrogen levels. So, it can be suggested that RPC has a greater effect on growth performance when supplemented in low-energy diets, and RPC addition can improve meat quality.
\end{abstract}

\section{Introduction}

Choline is a vitamin-like nutrient that functions as a biological methyl donor, in various reactions in the body thus playing a major role in energy metabolism. Although it can be synthesized by most animals, it is not produced in quantities sufficient to meet their requirements; as a result, external supplementation is often required. In ruminants, choline is rapidly degraded in the rumen (Sharma and Erdman, 1989); therefore, a rumen-protected form of choline has been developed for its safe delivery to the small intestine for absorption.

Rumen-protected choline (RPC) is commonly used in the diets of dairy cattle and dairy goats during the transition and early lactation periods, which are characterized by negative hormonal and energy balances, as it can prevent the development of fatty liver and improve milk yield and composition (Hartwell et al., 2000; Pinotti et al., 2008). However, the use of RPC in fattening goats has received little attention. In a recent study from Yangzhou University, 
China, it was shown that dietary supplementation with $0.25 \%$ RPC can promote weight gain in young lambs and improve meat quality (Li et al., 2015). In a subsequent study conducted in Egypt during summer it was found that RPC supplementation at $20 \mathrm{~g} / \mathrm{buck} /$ day in growing male Zaraibi goats under heat stress yielded the best growth performance, feed conversion and blood metabolite levels, and economic efficiency (Habeeb et al., 2017). These studies demonstrate that choline supplementation in growing sheep can effectively improve production performance and meat quality.

In the review on the effects of betaine on energy utilization in growing pigs, Cromwell et al. (1999) noted a greater response to betaine addition in animals fed lower-energy diets. As betaine is a chemical precursor of choline and has similar biological activity as a methyl donor, we suspected that choline may have a similar effect on fattening goats. We hypothesised that supplementation of RPC during fattening period would preferentially benefit goats fed low-energy diet, and expected an interaction of diet energy by RPC supplementation. The lack of ruminant feed has recently emerged to a serious issue in China, and has led to the development of some unconventional feeds from agricultural processing by-products for utilization in goat fattening. Most of these feeds have a common feature, i.e., highfibre and low-energy values, which reduce the diet energy content. As a result, addition of oil or fat-rich feed is required to increase the dietary energy level; however, this is restricted by animal metabolic physiology, and a more appropriate approach would improve the energy efficiency. This issue could be resolved if choline could achieve a greater response when fed with a lower-energy diet, as more energy would then be utilized for animal growth. Furthermore, as consumers are more concerned with meat quality, researchers now focus on the effects of feed additives in improving meat quality.

Therefore, we designed an experiment to evaluate the effects of RPC supplementation on the performance, meat quality and serum biochemical parameters of fattening goats at two different digestible energy levels. An interaction between dietary energy intake and RPC supplementation was expected.

\section{Material and methods}

The use of animals and experimental procedures were approved by the Jiangsu Academy of Agricultural Sciences Animal Care and Use Committee (China).

\section{Animals and experimental design}

The experiment was conducted at an experimental goat farm, in Suqian city, Jiangsu Province, China, from August to October 2018. In total, 40 six-month-old Cashmere goats, with an initial live body weight of $24.30 \pm 2.64 \mathrm{~kg}$, were randomly assigned to one of the four treatments, with 10 individuals per treatment. Diets were prepared according to the standard feed for meat-producing sheep and goats (MOA, 2014; NY/T 816-2004). The experiments were performed in a $2 \times 2$ factorial design consisting of two levels of RPC ( 0 or $0.2 \%)$ and either a low $(10.89 \mathrm{MJ} / \mathrm{kg})$ or high $(11.94 \mathrm{MJ} / \mathrm{kg})$ digestible energy (DE) content (Table 1). The RPC supplementation level was determined based on published literature and previous research results. The RPC, containing 25\% choline chloride, was obtained from Shanghai Mei Nong Biotechnology Co., Ltd (Shanghai, China).

Table 1. Four dietary treatments arranged in a $2 \times 2$ factorial design

\begin{tabular}{ll}
\hline RPC supplementation level, \% & Dietary DE level, MJ/kg \\
\hline 0 & 11.94 \\
& 10.89 \\
0.2 & 11.94 \\
& 10.89 \\
\hline
\end{tabular}

RPC - rumen-protected choline; DE - digestible energy

Animals were fed maize-soybean meal-based diets, and the different DE levels were obtained by replacing maize and soybean meal with whole cottonseed (Table 2).

The goats were housed in individual pens and were fed twice a day, at 8:00 and 17:00. Fresh water was available ad libitum. The experiment lasted for 70 days, with the first 10 days for acclimation and next 60 days as the formal experimental period. All goats were weighed at the beginning of the experiment, and then every 20 days till the end of the experiment. The offered and residual feeds were recorded daily. The average daily feed intake (ADFI), average daily gain $(\mathrm{ADG})$ and feed to gain ratio $(\mathrm{F} / \mathrm{G})$ were calculated to evaluate the production performance.

\section{Blood samples}

Blood samples were collected from the jugular vein at the end of the experiment. Samples were allowed to clot for $30 \mathrm{~min}$ and then centrifuged at $1125 \mathrm{~g}$ for $15 \mathrm{~min}$ to obtain the serum, which was stored at $-20^{\circ} \mathrm{C}$ for further analyses. The blood samples were analysed in the General Hospital of Nanjing Military Region (China), using Hita-chi 7020 blood biochemical analyser (Hitachi, Tokyo, Japan) 
Table 2. Ingredients and nutrient composition of the used diets, on a dry matter (DM) basis

\begin{tabular}{|c|c|c|c|c|c|}
\hline Ingredient, $\%$ of DM & High-energy diet & Low-energy diet & Nutrient composition & High-energy diet & Low-energy diet \\
\hline Maize (ground) & 26.00 & 16.50 & DM & 90.40 & 90.46 \\
\hline Whole cottonseed & 10.00 & 0 & $\mathrm{DE}, \mathrm{MJ} / \mathrm{kg}$ & 11.94 & 10.89 \\
\hline Soybean meal & 3.00 & 6.50 & $\mathrm{CP}, \% \mathrm{DM}$ & 14.03 & 14.02 \\
\hline Wheat bran & 8.00 & 8.00 & NDF, \% DM & 34.58 & 38.29 \\
\hline Soybean hull & 6.00 & 6.00 & $A D F, \% D M$ & 22.70 & 24.71 \\
\hline Sunflower meal & 4.00 & 4.00 & Ash, \% DM & 9.06 & 9.61 \\
\hline Maize germ meal & 5.00 & 12.00 & $\mathrm{Ca}, \% \mathrm{DM}$ & 0.92 & 0.92 \\
\hline Soybean straw & 8.00 & 17.00 & $\mathrm{P}, \% \mathrm{DM}$ & 0.42 & 0.42 \\
\hline Peanut straw & 20.00 & 20.00 & Price, Yuan/kg & 1.65 & 1.51 \\
\hline Alfalfa block & 5.00 & 5.00 & & & \\
\hline Yeast culture & 0.30 & 0.30 & & & \\
\hline Rice husk & 0.97 & 0.93 & & & \\
\hline $\mathrm{NaCl}$ & 0.60 & 0.60 & & & \\
\hline $\mathrm{CaHPO}_{4}$ & 0.42 & 0.25 & & & \\
\hline Limestone & 0.76 & 0.77 & & & \\
\hline Bentonite & 0.70 & 0.90 & & & \\
\hline Calcium propionate & 0.25 & 0.25 & & & \\
\hline $\mathrm{NaHCO}_{3}$ & 0.50 & 0.50 & & & \\
\hline Premix ${ }^{a}$ & 0.50 & 0.50 & & & \\
\hline Total & 100.00 & 100.00 & & & \\
\hline
\end{tabular}

a provided per kg of diet, IU: vit. A 4000 , vit. $\mathrm{D}_{3} 400$, vit. E 20 000; mg: FeSO $69.03, \mathrm{CuSO}_{4} 17.6, \mathrm{~K}_{2} \mathrm{SO}_{4} 31.70, \mathrm{ZnSO}_{4} 57.14, \mathrm{MnSO}_{4} 44.03$, $\mathrm{CoCl}_{2} 0.25, \mathrm{Na}_{2} \mathrm{SeO}_{3} 8.95$, monensin 6, $\mathrm{NaHCO}_{3} 740.91 ; \mathrm{DE}$ - digestible energy, $\mathrm{CP}$ - crude protein, NDF - neutral detergent fibre, $\mathrm{ADF}^{4}$ - acid detergent fibre

to detect for total bilirubin (T-Bili), total protein (TP), albumin (ALB), glucose (GLU), aspartate aminotransferase (AST), alanine aminotransferase (ALT) and blood urea nitrogen (BUN) contents.

\section{Carcass characteristics and meat quality}

At the end of the experiment, 12 randomlyselected goats (three per group) were slaughtered after a 24-h fasting period to determine the meat quality. The head, skin and visceral organs were removed immediately, and the live and carcass weights were recorded. The eye muscle area was calculated, and samples of musculus longissimus dorsi were collected from the region between the $12^{\text {th }}$ and $13^{\text {th }}$ vertebrae to evaluate the dripping loss, $\mathrm{pH}$, meat colour (45 min and $24 \mathrm{~h}$ ), cooking loss and shear force.

\section{Statistical analysis}

All data were analysed using the SPSS software (Version 12.0; IBM, Armonk, NY, USA). Data were expressed as mean and standard error of the mean (SEM) of the whole, the differences between groups were tested using one-way analysis of variance (ANOVA) with Duncan's multiple range test. Results with $P<0.05$ were considered statistically significant. Two-way ANOVA with generalized linear models was used to evaluate the main effects of RPC (with vs without), energy level (high vs low) and their interaction $(\mathrm{RPC} \times \mathrm{DE})$. Results with $P<0.05$ were considered significant, $P<0.01$ were extremely significant, and $0.05 \leq P<0.10$ indicated a tendency to differ.

\section{Results}

\section{Growth performance of fattening goats}

The effects of RPC supplementation in different energy level diets on growth performance are shown in Table 3. Both DE and RPC significantly affected the overall ADFI of goats $(P<0.05)$. The addition of RPC significantly improved the feed intake, particularly when applied with the low-energy diet, during days $40-60$ of the experiment $(P<0.05)$. In contrast, it had a negative effect on feed intake, when applied with the high-energy diet, during the $2^{\text {nd }}$ and $3^{\text {rd }}$ phases (days 20-60) of the experiment $(P>0.05)$. Regarding the ADG, the main effect of RPC supplementation became significant during days $20-40$ $(P<0.05)$. The ADG was significantly increased, by $60.88 \%$, in the group fed the low energy diet $(P<0.05)$ in comparison with an increase of only $5.71 \%$ in the high-energy diet group $(P>0.05)$ and a tendency of $\mathrm{RPC} \times \mathrm{DE}$ was observed $(P=0.06)$. 
Table 3. Effects of rumen-protected choline (RPC) at different dietary energy levels on growth performance

\begin{tabular}{|c|c|c|c|c|c|c|c|c|c|}
\hline \multirow{4}{*}{ Indices } & \multirow{4}{*}{ Time } & \multicolumn{4}{|l|}{$\mathrm{DE}, \mathrm{MJ} / \mathrm{kg}$} & \multirow{4}{*}{ SEM } & \multicolumn{3}{|c|}{ Main effects ${ }^{1}, P$-values } \\
\hline & & \multirow{2}{*}{\multicolumn{2}{|c|}{$\frac{11.87}{\mathrm{RPC}, \%}$}} & \multirow{2}{*}{\multicolumn{2}{|c|}{$\frac{10.53}{\text { RPC, } \%}$}} & & \multirow{3}{*}{ DE } & \multirow{3}{*}{ RPC } & \multirow{3}{*}{$\mathrm{RPC} \times \mathrm{DE}$} \\
\hline & & & & & & & & & \\
\hline & & 0 & 0.2 & 0 & 0.2 & & & & \\
\hline \multirow[t]{4}{*}{$\overline{\mathrm{ADFI}}$, g/day } & $0-20$ days & $1345.79^{a b^{*}}$ & $1432.63^{b^{*}}$ & $1250.00^{\mathrm{a}^{*}}$ & $1255.25^{\mathrm{a}^{*}}$ & 25.68 & $<0.01$ & 0.36 & 0.41 \\
\hline & 20-40 days & $1618.50^{a b^{\wedge}}$ & $1589.25^{\mathrm{a}^{\wedge}}$ & $1567.50^{\mathrm{a}^{\wedge}}$ & $1665.50^{\mathrm{b}^{\wedge}}$ & 11.96 & 0.58 & 0.14 & $<0.01$ \\
\hline & 40-60 days & $1692.50^{\mathrm{b \#}}$ & $1644.00^{b^{\wedge}}$ & $1490.00^{\mathrm{a}^{\wedge}}$ & $1718.25^{\wedge}$ & 16.4 & $<0.05$ & $<0.01$ & $<0.01$ \\
\hline & Overall & $1552.26^{b}$ & $1555.29^{b}$ & $1435.83^{a}$ & $1546.33^{b}$ & 14.26 & $<0.05$ & $<0.05$ & 0.06 \\
\hline \multirow[t]{4}{*}{ ADG, g/day } & $0-20$ days & $185.00^{*}$ & $167.75^{\star \wedge}$ & $175.56^{*}$ & $216.25^{*}$ & 11.32 & 0.40 & 0.61 & 0.22 \\
\hline & 20-40 days & $205.75^{*}$ & $217.50^{b^{*}}$ & $135.00^{\mathrm{a}^{*}}$ & $217.19^{b^{*}}$ & 10.38 & 0.06 & $<0.05$ & 0.06 \\
\hline & $40-60$ days & $182.75^{*}$ & $153.50^{\mathrm{ab}^{\wedge}}$ & $150.56^{\mathrm{ab}^{\mathrm{a}}}$ & $132.19^{\mathrm{a}^{\wedge}}$ & 6.35 & $<0.05$ & $<0.05$ & 0.65 \\
\hline & Overall & $191.17^{b}$ & $179.58^{\mathrm{ab}}$ & $153.70^{\mathrm{a}}$ & $188.54^{\mathrm{ab}}$ & 6.16 & 0.24 & 0.33 & 0.06 \\
\hline Feed:gain ratio & Overall & 8.31 & 9.02 & 9.55 & 8.59 & 0.27 & 0.47 & 0.82 & 0.14 \\
\hline
\end{tabular}

$\mathrm{DE}$ - digestible energy; SEM - standard error of the mean; ${ }^{1} P$-values of main effect of DE = high energy vs low energy, $P$-values of main effect of $\mathrm{RPC}=-\mathrm{RPC}$ vs + RPC, $P$-values of cross effect of RPC $\times D E$ interaction; $A D F I$ - average daily feed intake; ADG - average daily gain; ${ }^{\text {ab }}$ - means with different superscripts in the same row are significantly different at $P<0.05$; ${ }^{* \#}$ - means with different signs within the same column are significantly different at $P<0.05$; overall was not included in the statistics

However, the main effect of DE became significant during days $40-60(P<0.05)$, while the main effect of RPC weakened, showing a tendency to decrease the ADG $(P=0.05)$. Furthermore, for the overall experimental period, the effect of neither DE nor RPC on ADG was significant $(P>0.05)$. Nevertheless, it was found that RPC supplementation with low-energy diet was more effective and enhanced the ADG by $22.67 \%$ in comparison with a reduction of $6.06 \%$ in the high-energy diet.

Differences within columns for each trait indicated ADFI of the four groups in the $2^{\text {nd }}$ stage (days 20-40) were all significantly higher than in the $1^{\text {st }}$ stage (days $\left.0-20\right)(P<0.05)$, the largest increase was in low-energy diet with RPC addition group $(32.8 \%)$. During the $3^{\text {rd }}$ stage (days 40-60), the ADFI was further significantly enhanced with the increase of dietary energy level $(P<0.05)$, but the remaining groups had no significant difference with $2^{\text {nd }}$ stage. Values of ADG in both the high-energy without RPC group and low-energy without RPC group did not significantly differ in the three stages $(P>0.05)$, but after adding RPC, both the lowenergy group and the high-energy group showed a significant decrease in ADG in the $3^{\text {rd }}$ stage (40 to 60 days) $(P<0.05)$. The overall $\mathrm{F} / \mathrm{G}$ was not significantly affected by DE, RPC or their interaction $(P>0.05)$. However, the effect of RPC supplementation on $\mathrm{F} / \mathrm{G}$ was consistent with that on ADG, i.e., a greater response was observed for RPC supplementation in low-energy diet, resulting in a $10.05 \%$ reduction in $\mathrm{F} / \mathrm{G}$ as compared with low-and high-energy diets without RPC supplementation.

\section{Slaughter performance of fattening goats}

The dressing percentage and eye muscle area were not significantly affected by RPC supplementation, dietary energy level or their interaction $(P>0.05)$ (Table 4).

\section{Meat quality of fattening goats}

As shown in Table 5, dietary energy had a significant main effect on meat shearing force $(P<0.05)$, which increased in line with increasing dietary energy level without RPC supplementation $(P<0.05)$. Dietary energy enhancement also tended to increase meat cooking loss $(0.05<P<0.10)$. Furthermore, RPC supplementation had a significant

Table 4. Effects of rumen-protected choline (RPC) at different dietary energy levels on slaughter performance

\begin{tabular}{|c|c|c|c|c|c|c|c|c|}
\hline \multirow{4}{*}{ Indices } & \multicolumn{4}{|c|}{$\mathrm{DE}, \mathrm{MJ} / \mathrm{kg}$} & \multirow{4}{*}{ SEM } & \multicolumn{3}{|c|}{ Main effects ${ }^{1}, P$-values } \\
\hline & \multirow{2}{*}{\multicolumn{2}{|c|}{$\frac{11.87}{\mathrm{RPC}, \%}$}} & \multirow{2}{*}{\multicolumn{2}{|c|}{$\frac{10.53}{\mathrm{RPC}, \%}$}} & & \multirow{3}{*}{ DE } & \multirow{3}{*}{ RPC } & \multirow{3}{*}{$\mathrm{RPC} \times \mathrm{DE}$} \\
\hline & & & & & & & & \\
\hline & 0 & 0.2 & 0 & 0.2 & & & & \\
\hline Weight before slaughter, $\mathrm{kg}$ & 38.88 & 37.37 & 36.90 & 37.04 & 0.65 & 0.44 & 0.64 & 0.57 \\
\hline Carcass weight, $\%$ & 17.67 & 17.75 & 16.39 & 17.06 & 0.46 & 0.36 & 0.72 & 0.78 \\
\hline Dressing percentage, $\%$ & 45.44 & 47.39 & 44.37 & 46.02 & 0.65 & 0.38 & 0.21 & 0.91 \\
\hline Eye muscle area, $\mathrm{cm}^{2}$ & 15.67 & 16.63 & 15.81 & 15.96 & 0.30 & 0.39 & 0.13 & 0.50 \\
\hline
\end{tabular}

$\mathrm{DE}$ - digestible energy; SEM - standard error of the mean; ${ }^{1} P$-values of main effect of $\mathrm{DE}=$ high energy vs low energy, $P$-values of main effect of RPC $=-\mathrm{RPC}$ vs $+\mathrm{RPC}, P$-values of cross effect of RPC $\times D E$ interaction 
Table 5. Effects of rumen-protected choline (RPC) at different dietary energy level on meat quality

\begin{tabular}{|c|c|c|c|c|c|c|c|c|}
\hline \multirow{4}{*}{ Indices } & \multicolumn{4}{|c|}{$\mathrm{DE}, \mathrm{MJ} / \mathrm{kg}$} & \multirow{4}{*}{ SEM } & \multicolumn{3}{|c|}{ Main effects ${ }^{1}, P$-values } \\
\hline & \multirow{2}{*}{\multicolumn{2}{|c|}{$\begin{array}{l}11.87 \\
\mathrm{RPC}, \%\end{array}$}} & \multirow{2}{*}{\multicolumn{2}{|c|}{$\frac{10.53}{\mathrm{RPC}, \%}$}} & & \multirow{3}{*}{$\mathrm{DE}$} & \multirow{3}{*}{$\mathrm{RPC}$} & \multirow{3}{*}{$\mathrm{RPC} \times \mathrm{DE}$} \\
\hline & & & & & & & & \\
\hline & 0 & 0.2 & 0 & 0.2 & & & & \\
\hline Drip loss, $\%$ & 4.28 & 3.98 & 4.35 & 4.23 & 0.18 & 0.71 & 0.61 & 0.83 \\
\hline Cook loss, $\%$ & $32.93^{b}$ & $31.65^{b}$ & $32.63^{b}$ & $28.07^{a}$ & 0.72 & 0.08 & $<0.05$ & 0.14 \\
\hline Shear force, \% & $58.58^{a}$ & $56.45^{\mathrm{ab}}$ & $55.51^{b}$ & $55.15^{b}$ & 0.51 & $<0.05$ & 0.13 & 0.27 \\
\hline \multicolumn{9}{|l|}{ Meat colour } \\
\hline $\mathrm{L}_{45 \min }$ & 36.52 & 38.15 & 37.50 & 36.97 & 0.92 & 0.96 & 0.80 & 0.62 \\
\hline$a_{45 \min }$ & 17.44 & 16.71 & 18.66 & 15.46 & 0.72 & 0.99 & 0.22 & 0.43 \\
\hline $\mathrm{b}_{45 \min }$ & 5.46 & 5.59 & 6.32 & 5.24 & 0.29 & 0.70 & 0.46 & 0.36 \\
\hline \multicolumn{9}{|l|}{ Meat colour } \\
\hline $\mathrm{L}_{24 \mathrm{~h}}$ & 40.38 & 40.83 & 40.95 & 41.93 & 0.80 & 0.66 & 0.71 & 0.89 \\
\hline$a_{24 h}$ & 13.74 & 14.62 & 16.29 & 14.86 & 0.48 & 0.17 & 0.78 & 0.25 \\
\hline$b_{24 h}$ & 7.27 & 7.07 & 8.30 & 8.30 & 0.38 & 0.21 & 0.90 & 0.90 \\
\hline $\mathrm{pH}_{45 \min }$ & 6.27 & 6.40 & 6.18 & 6.11 & 0.06 & 0.17 & 0.82 & 0.41 \\
\hline $\mathrm{pH}_{24 \mathrm{~h}}$ & 5.83 & 5.81 & 5.82 & 5.81 & 0.04 & 0.96 & 0.91 & 0.96 \\
\hline
\end{tabular}

$\mathrm{DE}$ - digestible energy; SEM - standard error of the mean; ${ }^{1} P$-values of main effect of $\mathrm{DE}=$ high energy vs low energy, $P$-values of main effect of RPC = -RPC vs + RPC, $P$-values of cross effect of RPC $\times D E$ interaction; $L-$ brightness, $a$ - redness, $b$ - yellowness; ${ }^{\text {ab }}-$ means with different superscripts in the same row are significantly different at $P<0.05$

Table 6. Effects of rumen-protected choline (RPC) at different dietary energy levels on blood biochemical indices

\begin{tabular}{|c|c|c|c|c|c|c|c|c|}
\hline \multirow{4}{*}{ Indices } & \multicolumn{4}{|c|}{$\mathrm{DE}, \mathrm{MJ} / \mathrm{kg}$} & \multirow{4}{*}{ SEM } & \multicolumn{3}{|c|}{ Main effects ${ }^{1}, P$-values } \\
\hline & \multirow{2}{*}{\multicolumn{2}{|c|}{$\begin{array}{l}11.87 \\
\mathrm{RPC}, \%\end{array}$}} & \multirow{2}{*}{\multicolumn{2}{|c|}{$\frac{10.53}{\mathrm{RPC}, \%}$}} & & \multirow{3}{*}{$\mathrm{DE}$} & \multirow{3}{*}{ RPC } & \multirow{3}{*}{$\mathrm{RPC} \times \mathrm{DE}$} \\
\hline & & & & & & & & \\
\hline & 0 & 0.2 & 0 & 0.2 & & & & \\
\hline Total bilirubin, $\mu \mathrm{mol} / /$ & 14.05 & 12.67 & 13.95 & 14.17 & 0.49 & 0.50 & 0.58 & 0.44 \\
\hline Total protein, g/l & 69.05 & 65.76 & 69.86 & 69.29 & 0.69 & 0.11 & 0.15 & 0.31 \\
\hline Albumin, g/l & $31.99^{\mathrm{ab}}$ & $35.50^{b}$ & $29.30^{\mathrm{a}}$ & $33.31^{\mathrm{ab}}$ & 0.77 & 0.08 & $<0.01$ & 0.85 \\
\hline Glucose, $\mathrm{mmol} / \mathrm{l}$ & $2.77^{\mathrm{a}}$ & $3.09^{b}$ & $3.05^{\mathrm{ab}}$ & $3.11^{\mathrm{b}}$ & 0.05 & 0.15 & 0.07 & 0.21 \\
\hline Aspartate aminotransferase, $\mathrm{U} / \mathrm{l}$ & 54.40 & 55.03 & 57.56 & 52.31 & 2.47 & 0.97 & 0.67 & 0.59 \\
\hline Alanine, U/I & 54.62 & 57.67 & 63.83 & 59.27 & 1.99 & 0.19 & 0.85 & 0.35 \\
\hline Blood urea nitrogen, $\mathrm{mmol} / \mathrm{l}$ & $17.52^{b}$ & $15.64^{\mathrm{ab}}$ & $15.48^{\mathrm{ab}}$ & $13.78^{a}$ & 0.42 & $<0.05$ & $<0.05$ & 0.90 \\
\hline
\end{tabular}

$\mathrm{DE}$ - digestible energy; SEM - standard error of the mean; ${ }^{1} P$-values of main effect of DE = high energy vs low energy, $P$-values of main effect of $\mathrm{RPC}=-\mathrm{RPC}$ vs $+\mathrm{RPC}, P$-values of cross effect of RPC $\times D E$ interaction; ${ }^{\text {ab }}$ - means with different superscripts in the same row are significantly different at $P<0.05$

main effect on cooking loss $(P<0.05)$, which was decreased by $13.97 \%$ in the low-energy diet group $(P<0.05)$ in comparison with a decrease of only $3.89 \%$ in the high-energy diet group $(P>0.05)$. Meat colour and $\mathrm{pH}$ values measured $45 \mathrm{~min}$ and $24 \mathrm{~h}$ after slaughter were not affected by RPC or DE $(P>0.05)$, and no interaction effect of RPC $\times \mathrm{DE}$ could be detected for any meat quality parameter tested $(P>0.05)$.

\section{Blood biochemical parameters of fattening goats}

Blood biochemical parameters are listed in Table 6. RPC exerted a significant effect on ALB $(P<0.05)$ and BUN $(P<0.05)$, and had a tendency to affect GLU $(0.05<P<0.1)$. DE had a significant effect on BUN $(P<0.05)$, and a tendency to affect
ALB $(0.05<P<0.1)$. However, their interaction did not have any significant effect $(P>0.05)$. ALB concentration was increased by RPC supplementation at both energy levels $(P>0.05)$. GLU concentration was significantly improved by RPC supplementation in high-energy diets $(P<0.05)$. BUN concentration decreased after RPC supplementation at both dietary energy levels $(P>0.05)$. No significant effect of RPC $\times$ DE interaction was detected for any blood biochemical parameter $(P>0.05)$.

\section{Discussion}

Effect on growth performance. In numerous studies it was reported an increase in dry matter intake (DMI) of dairy cows supplemented with RPC 
(Grummer, 2012; Soltan et al., 2012), whereas in some studies no effect of RPC on the DMI of cows and goats was found (Bryant et al., 1999; Garg et al., 2012). The effect of choline on animal feed intake is dependent on many factors, such as its purity, the amount added, protection form and animal body condition. In the present study, DMI was significantly increased by RPC addition, which may be attributed to the improvement of body metabolism, resulting in higher feed intake. Supplementation of RPC increases milk production in cows, improves milk components and increases ADG in heifers, and these effects are independent of the prepartum caloric intake (Zenobi et al., 2018). However, in the present study, we observed a tendency of the RPC $\times \mathrm{DE}$ interaction to affect goat growth performance. The effect of RPC was greater when supplemented in low-energy diets. A similar response was observed by Habeeb et al. (2017), who reported the best growth, feed conversion, blood metabolite levels and economic efficiency in Zaraibi goats fed diets supplemented with RPC, particularly subjected to heat stress during the Egyptian summer. We assumed that RPC supplementation may have a greater effect under stress conditions, such as high temperature or low dietary energy. In a review of the effects of betaine on energy utilization in growing pigs it was also documented that betaine can retain water inside the cell at lower-energy expenditure, thus reducing the energy requirements for cellular maintenance, allowing more energy to be used for animal growth (Lipiński et al., 2012). The addition of betaine can increase the energy availability, thus improving the efficiency of low-energy diets (Schrama et al., 2003). Betaine is a product of choline oxidation, and thus, these findings support our results; however, the specific mechanism requires further investigations.

Comprising the results of three stages of the study, it can be seen that the addition of RPC had a significant effect on the increase of ADFI especially in low-energy diets. The addition of RPC in lowenergy diets could significantly increase the ADG during the first two stages of the experiment, but the effect of RPC was weakened in the last stage of the experiment. It is consistent with the main effect analysis result (presented in Table 3 ). The main effect of RPC on ADG was weakened at the last stage of the experiment. Therefore, RPC is more suitable to add early in the low-energy diets, and its mechanism needs further studies.

Effect on slaughter performance and meat quality. RPC addition did not have a significant influence on slaughter performance. However, it resulted in lower drip loss, cook loss and shearing force, thus exhibiting a tendency to improve meat quality. This is consistent with the findings of $\mathrm{Li}$ et al. (2015). Choline occurs in the cell membranes as lecithin, and improves the structural integrity of cell membranes, thereby enhancing meat quality. Choline can be also transformed into phosphatidylcholine, which is an important component of the cell membrane. The ratio of phosphatidylcholine to phosphatidylethanolamine is a key regulator of membrane integrity, and a decrease in this ratio can even lead to cell death (Yen et al., 1999). Thus, RPC supplementation may increase phosphatidylcholine synthesis, thereby increasing the ratio of phosphatidylcholine to phosphatidylethanolamine, resulting in improved cell integrity, and lower drip loss and shearing force of meat.

Effects on haematological parameters. ALB and TP indicate immunity functions in animals. Our results are consistent with a previous report of increased ALB percentage in RPC-supplemented cows (Rahmani et al., 2014). The increase in GLU following RPC supplementation may be attributed to choline-mediated increase in glucose transport to provide energy for peptide synthesis, as reported by Rahmani et al. (2014). In the present study, any effect of RPC supplementation on AST and ALT was found, indicating that the goats were in good health. This result was consistent with the findings of Habeeb et al. (2017), who did not observe any effect of RPC supplementation on plasma AST and ALT in growing male Zaraibi goats. Serum urea nitrogen is an important indicator of nitrogen metabolism in the body. Decreased serum urea nitrogen indicates negative changes in protein decomposition and an increase in protein precipitation. We observed a significant reduction of BUN following RPC addition, whereas an increase in the dietary energy level significantly increased the BUN concentration. This indicates that RPC supplementation can increase the utilization of protein in the feed, which cannot be achieved by an increase in the dietary energy level alone.

\section{Conclusions}

An improvement in the growth performance, especially in the early fattening period, of fattening goats fed a low-energy diet with rumen-protected choline (RPC) addition was observed. Consumption of such a diet resulted in lower drip loss, cook loss and shearing force of meat, and showed a tendency to improve meat quality. So, using RPC as a feed 
additive in fattening goat feeding is effective and has economic advantages. It was shown that lowenergy diet has a positive impact on goat production performance (close to high-energy diets productive results), and results in good meat quality.

\section{Acknowledgements}

Zhi-peng Zhang, Xiao-han Zhang, Mei-juan Meng, Li-peng Gao, and Kai Zhang helped ensure proper daily care of goats and assisted in sample collection. The experiment was partially supported by Shanghai Mei Nong Biotechnology Co., Ltd.

This study was supported by the Project of Special funds for scientific research in non-profit industries (agriculture) (Grant No. 201103050) and the Key Laboratory for Crop and Animal Integrated Farming of Ministry of Agriculture and Rural Affairs Open Subject (Grant No. 201904).

\section{References}

Bryant T.C., Rivera J.D., Galyean M.L., Duff G.C., Hallford D.M., Montgomery T.H., 1999. Effects of dietary level of ruminally protected choline on performance and carcass characteristics of finishing beef steers and on growth and serum metabolites in lambs. J. Anim. Sci. 77, 2893-903, https://doi. org/10.2527/1999.77112893x

Cromwell G.L., Lindemann M.D., Randolph J.R., Monegue H.J., Laurent K.M., Parker G.R., 1999. Efficacy of betaine as a carcass modifier in finishing pigs fed normal and reduced energy diets. J. Anim. Sci. 77, Suppl. 1, 179

Garg M.R., Sherasia P.L., Bhanderim B.M., 2012. Effect of supplementing bypass fat with and without rumen protected choline chloride on milk yield and serum lipid profile in jaffarabadi buffaloes. Buffalo Bull. 31, 91-98

Grummer R.R., 2012. Choline: a limiting nutrient for transition dairy cows. Proceedings of the Cornell Nutrition Conference. Cornell University. Syracuse, NY (USA), pp. 22-27

Hartwell J.R., Cecava M.J., Donkin S.S., 2000. Impact of dietary rumen undegradable protein and rumen-protected choline on intake, peripartum liver triacylglyceride, plasma metabolites and milk production in transition dairy cows. J. Dairy Sci. 83, 2907-2917, https://doi.org/10.3168/jds.S0022-0302(00)75191-5
Habeeb A.A.M., Gad A.E., Atta M.A.A., Abdel-hafez M.A.M., 2017. Evaluation of rumen-protected choline additive to diet on productive performance of male Zaraibi growing goats during hot summer season in Egypt. Trop. Anim. Health Prod. 49, 1107-1115, https://doi.org/10.1007/s11250-017-1292-x

Li H.W., Wang H.R., Yu L.H., Wang M.Z., Liu S.M., Sun L.S., Chen Q., 2015. Effects of supplementation of rumen-protected choline on growth performance, meat quality and gene expression in longissimus dorsi muscle of lambs. Arch. Anim. Nutr. 69, 340-350, https://doi.org/10.1080/1745039X.2015.1073001

Lipiński K., Szramko E., Jeroch H., Matusevičius P., 2012. Effects of betaine on energy utilization in growing pigs - a review. Ann. Anim. Sci. 12, 291-300, https://doi. org/10.2478/v10220-012-0024-4

MOA (Ministry of Agriculture, China), 2004. Feeding Standard of MeatProducing Sheep and Goats of China (NY/T 816-2004)

Pinotti L., Campagnoli A., D' Ambrosio F. et al., 2008. Rumen-protected choline and vitamin $E$ supplementation in periparturient dairy goats: effects on milk production and folate, vitamin $B_{12}$ and vitamin E status. Animal 2, 1019-1027, https://doi. org $/ 10.1017 / \mathrm{S} 1751731108002103$

Rahmani M., Dehghan-banadaky M., Kamalyan R. et al., 2014. Effects of feeding rumen-protected choline and vitamin $E$ on serum protein fractions, total thiol molecules and total antioxidant capacity in early lactating dairy cows. Global J. Anim. Sci. Res. 2, 337-344

Sharma B.K., Erdman R.A., 1989. In vitro degradation of choline from selected feedstuffs and choline supplements. J. Dairy Sci. 72, 2772-2776, https://doi.org/10.3168/jds.S00220302(89)79421-2

Schrama J.W., Heetkamp M.J., Simmins P.H., Gerrits W.J.J., 2003. Dietary betaine supplementation affects energy metabolism of pigs. J. Anim. Sci. 81, 1202-1211, https://doi. org/10.2527/2003.8151202x

Soltan M.A., Mujalli A.M., Mandour M.A., Abeer M.E., 2012. Effect of dietary rumen protected methionine and/or choline supplementation on rumen fermentation characteristics and productive performance of early lactating cows. Pakistan J. Nutr. 11, 221-230, https://doi.org/10.3923/pjn.2012.221.230

Yen C.E., Mar M., Zeisel S.H., 1999. Choline deficiency-induced apoptosis in pc12 cells is associated with diminished membrane phosphatidylcholine and sphingomyelin, accumulation of ceramide and diacylglycerol, and activation of a caspase. FASEB J. 13, 135-142, https://doi.org/10.1096/ fasebj.13.1.135

Zenobi M.G., Gardinal R., Zuniga J.E., Dias A.L.G. et al., 2018. Effects of supplementation with ruminally protected choline on performance of multiparous Holstein cows did not depend upon prepartum caloric intake. J. Dairy Sci. 101, 1088-1110, https://doi.org/10.3168/jds.2017-13327 